\title{
Reacciones alérgicas a betalactámicos en pediatría: recomendaciones para su diagnóstico y tratamiento
} Allergic Reactions to Betalactams in Pediatrics: Recommendations
for diagnosis and treatment

Comité Nacional de Alergia ${ }^{a}$

\section{RESUMEN}

Las reacciones alérgicas a antibióticos betalactámicos son eventos adversos no explicables por su acción farmacológica, sino mediados por un mecanismo inmunológico. Son la causa más frecuente de alergia a los fármacos en el mundo. Incluyen penicilinas, cefalosporinas, carbapenems, monobactámicos, cefems (moxalactam, cefoxitina) y ácido clavulánico. Pueden producir reacciones alérgicas inmediatas, aceleradas o tardías. Su metodología diagnóstica es compleja y se realiza por etapas bien definidas. Es muy importante su diagnóstico oportuno, debido al riesgo que implican y a los costos asociados, que demandan el uso alternativo de otros antibióticos que los reemplacen.

Palabras clave: hipersensibilidad a las drogas, beta-lactámicos, penicilina $G$, ampicilina, anafilaxia.

\begin{abstract}
The Allergic Reactions to Betalactam Antibiotics are adverse events that cannot be explained by their pharmacological action, but mediated by an immunological mechanism. They are the most frequent cause of drug allergy in the world. Betalactam antibiotics include penicillins, cephalosporins, carbapenems, monobactams, cefems (moxalactam, cefoxitin), and clavulanic acid. They can produce immediate, accelerated or delayed allergic reactions. Its diagnostic methodology is complex and it is carried out by well-defined stages. Its timely diagnosis is very important due to the risk involved and the associated costs that demand the alternative use of other antibiotics that supplant them.
\end{abstract}

Key words: drug hypersensitivity, beta-lactams, penicillin $G$, ampicillin, anaphylaxis.

http: / / dx.doi.org/10.5546/ aap.2019.S24

Cómo citar: Comité Nacional de Alergia. Reacciones alérgicas a betalactámicos en pediatría: recomendaciones para su diagnóstico y tratamiento. Arch Argent Pediatr 2019;117 Supl 1:S24-S36.

\section{GLOSARIO}

AGEP: exantema pustuloso generalizado agudo.

AINE: antiinflamatorios no esteroideos.

$C D$ : cluster of differentiation (grupo de diferenciación).

DRESS: exantema cutáneo con eosinofilia y síntomas sistémicos. ELISA: ensayo por inmunoabsorción ligado a enzimas.

EMF: exantema medicamentoso fijo. IgG: inmunoglobulina G.

IgE: inmunoglobulina E. IgM: inmunoglobulina $\mathrm{M}$. FEIA: fluoroenzymoinmunoensayo MDM: determinantes menores.

NET: necrólisis epidérmica tóxica.
Correspondencia: Dra. Andrea Irene Mariño: aimarino1966@ yahoo.com.ar

Financiamiento: Ninguno.

Conflicto de intereses: Ninguno que declarar.

Recibido: 9-9-2018 Aceptado: 12-10-2018
Miembros del Comité Nacional de Alergia de la Sociedad Argentina de Pediatría: Dra. Andrea I. Mariño*, Dra. María P. Sarraquigne, Dra. Gloria Bandín, Dr. Claudio Agüero, Dr. Ricardo Saranz, Dr. Mauricio Colella, Dra. Karina López, Dra. Betina Menéndez Porfilio, Dr. Fabio Orellano, Dra. Viviana Seisdedos, Dr. Alejandro Lozano, Dr. Raúl Boudet, Dr. Victor Skrie, Dra. Patricia Dayan, Dr. Juan M. Suárez, Dra. María E. Gervasoni, Dra. Marcela García, Dr. Dante Maldonado, Dra. Mónica Mata Rufolo, Dr. Antonio Sánchez Segovia, Dr. Aldo Cavallo, Dr. Adolfo Blanco, Dr. Claudio Parisi, Dr. Martín Bózzola, Dra. Renée Claeys, Dra. Nilda Daguerre, Dr. Horacio González, Dra. Alicia Lacher, Dra. María C. Cassaniti, Dra. Irene Aráoz, Dr. Julio Orellana, Dra. María E. Cáceres, Dr. Roque Arnolt, Dra. Elsa Mindel y Dra. Raquel Rodríguez.

* Unidad de Alergia del Hospital Municipal de Agudos Dr. Leónidas Lucero, de la ciudad de Bahía Blanca, asesora del Comité Nacional de Alergia de la Sociedad Argentina de Pediatría (SAP). 
PCLI: prueba cutánea de lectura inmediata.

PI: pruebas intradérmicas.

PPC: pruebas de provocación controlada.

PPL: determinantes mayores (bencilpeniciloilpolilisina).

RALB: reacciones alérgicas a betalactámicos.

RAM: reacciones adversas a medicamentos.

RALM: reacciones alérgicas a medicamentos.

RAST: radio allergo sorbent test.

RHS: reacciones de hipersensibilidad.

SSJ: síndrome de Stevens-Johnson.

TAB: test de activación de basófilos.

TPA: test del parche atópico.

TPO: test de provocación oral.

TTL: test de transformación linfocitaria.

UTIP: Unidad de Terapia Intensiva Pediátrica.

VIH: virus de la inmunodeficiencia adquirida

humana.

VEB: virus de Epstein-Barr.

HLA: antígenos leucocitarios humanos.

Este documento tiene como objetivo relevar información actualizada sobre las reacciones alérgicas a antibióticos betalactámicos (RALB) y brindar al pediatra herramientas diagnósticas y terapéuticas de abordaje integral para evitar riesgos y mejorar la calidad de vida de los niños alérgicos a los antibióticos betalactámicos.

\section{INTRODUCCIÓN}

Las reacciones adversas a medicamentos (RAM) se definen como cualquier efecto perjudicial o no deseado que ocurra tras la administración de una dosis medicamentosa normalmente utilizada en la especie humana para la profilaxis, el diagnóstico y/o el tratamiento de la enfermedad o para la modificación de una función biológica. ${ }^{1}$ Pueden clasificarse en 6 tipos (Tabla 1).

Ubicadas dentro de las RAM, las reacciones alérgicas a medicamentos (RALM) son eventos adversos no explicables por su acción farmacológica, sino mediados por un mecanismo inmunológico. Se caracterizan por su especificidad y reproducción con la reexposición al fármaco, ${ }^{2}$ y los mecanismos fisiopatológicos involucrados responden a la clasificación de Gell y Coombs ${ }^{3,4}$ de las reacciones de hipersensibilidad (RHS) (Figura 1).

\section{EPIDEMIOLOGÍA}

La alergia a medicamentos es, en la actualidad, el tercer motivo de consulta más frecuente en alergología, solo superado por el asma y la rinitis, y se constituye en un desafío en cuanto a la explicación de sus mecanismos inmunológicos y a la validación de nuevos métodos de estudio para su diagnóstico certero. ${ }^{4}$

Se estima que más del $70 \%$ de la población puede verse afectada por una RAM, pero solo son de tipo alérgico el $15 \%$ de ellas, ${ }^{5}$ aunque se sabe que la mayoría de estos estudios epidemiológicos se han llevado a cabo en el ámbito de la internación hospitalaria. ${ }^{6-8} \mathrm{Si}$ bien la percepción de alergia a medicamentos puede parecer mayor, se estima que la prevalencia en la población pediátrica se halla por debajo del 0,6\%.7 Según datos de Alergológica 2005, los niños representaron el 9,8\% de los pacientes que consultaron por alergia a fármacos, $\mathrm{y}$, de los pacientes remitidos por sospecha de hipersensibilidad a fármacos, este diagnóstico solo se confirmó en el $12 \%$ de los pacientes pediátricos estudiados. ${ }^{8}$

En pediatría, el $80 \%$ de los derivados para

TABLA 1. Clasificación de reacciones adversas a medicamentos, Rawling y Thompson, Page y Colb, Edwards y Aronson ${ }^{1}$

\begin{tabular}{|c|c|c|c|c|c|c|}
\hline Tipos & $\begin{array}{c}\text { A } \\
\text { Aumentadas } \\
70-90 \% \\
\text { predecibles } \\
\end{array}$ & $\begin{array}{c}\text { B } \\
\text { Bizarras } \\
\text { 10-30 \% } \\
\text { impredecibles }\end{array}$ & $\begin{array}{c}\text { C } \\
\text { Continuas }\end{array}$ & $\begin{array}{c}\text { D } \\
\text { Demoradas }\end{array}$ & $\begin{array}{c}\mathrm{E} \\
\text { Interrupción } \\
\text { de uso }\end{array}$ & $\begin{array}{c}\text { F } \\
\text { Foráneas }\end{array}$ \\
\hline Mecanism & $\begin{array}{c}\text { Dependiente } \\
\text { de la dosis }\end{array}$ & $\begin{array}{c}\text { Independiente } \\
\text { de la dosis }\end{array}$ & $\begin{array}{l}\text { Tratamiento } \\
\text { prolongado }\end{array}$ & $\begin{array}{c}\text { Efectos retardados: } \\
\text { mutagénesis, } \\
\text { inmunotoxicidad, } \\
\text { carcinogénesis y } \\
\text { teratogénesis }\end{array}$ & $\begin{array}{l}\text { Síndrome de } \\
\text { abstinencia y } \\
\text { efectos rebote } \\
\text { al suprimir } \\
\text { la medicación }\end{array}$ & $\begin{array}{c}\text { Causada por } \\
\text { agentes ajenos al } \\
\text { principio activo: } \\
\text { excipientes, } \\
\text { contaminantes }\end{array}$ \\
\hline Ejemplos & $\begin{array}{l}\text { Sobredosificación, } \\
\text { efectos colaterales y } \\
\text { secundarios }\end{array}$ & $\begin{array}{c}\text { Intolerancia } \\
\text { Idiosincrasia } \\
\text { Hipersensibilidad }\end{array}$ & $\begin{array}{c}\text { Discinesia } \\
\text { tardía por } \\
\text { neurolépticos }\end{array}$ & $\begin{array}{c}\text { Carcinoma de } \\
\text { células claras en } \\
\text { hijas de mujeres } \\
\text { que usaron } \\
\text { dietilestilbestrol }\end{array}$ & $\begin{array}{l}\text { Insuficiencia } \\
\text { suprarrenal } \\
\text { por suspensión } \\
\text { de corticoides }\end{array}$ & \\
\hline
\end{tabular}


estudio son menores de 3 años; suelen presentar cuadros de exantema morbiliforme o urticaria tras el uso de antibióticos, generalmente, betalactámicos, en el tratamiento de otitis, faringoamigdalitis, infecciones urinarias, que resultan difíciles de distinguir de las manifestaciones clínicas de la propia infección en curso.

Los antibióticos betalactámicos son la causa más frecuente de alergia a medicamentos en el mundo; su prevalencia oscila entre el $5 \%$ y el $10 \%$ en la población general, ${ }^{9}$ y tienen una frecuencia aproximada del $81 \%$ de todas las reacciones alérgicas a medicamentos en niños. ${ }^{7}$ En estudios en la población infantil, la prevalencia de reacciones reportadas a betalactámicos es del 1,7\% al 5,2\%. La amoxicilina representa el $1,4 \%$ de las causas; otras penicilinas, el 1,2\%, y cefalosporinas, el 0,7 \%. ${ }^{10-12}$

La amoxicilina ocupa el primer lugar como la causa más reportada en niños, con una prevalencia del $65 \%$, y los antiinflamatorios no esteroideos (AINE), con el $13 \%$, la segunda. ${ }^{7,9}$ Diferentes trabajos expresan que las manifestaciones clínicas son menos graves en niños, al igual que es menor la frecuencia de anafilaxia. ${ }^{10}$

Los factores de riesgo en el desarrollo de alergia a medicamentos ${ }^{12-18}$ son los siguientes:

- Edad adulta: entre 20 y 49 años.

- Más frecuente en las mujeres.

- Exposición recurrente a los mismos fármacos, ya que representa un riesgo de sensibilización, que puede ocurrir al mismo fármaco o a otro de reactividad cruzada. Además, a mayor dosis y frecuencia de utilización, mayor es la posibilidad de sensibilización por un mecanismo inmunológico, ${ }^{14}$ como puede observarse, por ejemplo, en pacientes con fibrosis quística.

- Frecuencia de administración y ruta de exposición: número de dosis administradas, mayor duración del tratamiento, tratamientos discontinuos y múltiples son causas comunes de sensibilización. ${ }^{14}$ La aplicación tópica de penicilina y derivados es altamente inmunogénica. ${ }^{12}$

- Reacciones alérgicas previas a medicamentos: cuando se da en medicamentos no relacionados químicamente, podría deberse a un aumento de la capacidad de respuesta de sus células $\mathrm{T}$ frente a fármacos.

- Infección por virus de la inmunodeficiencia humana (VIH) (sulfamidas), virus de EpsteinBarr (VEB) y herpes virus (aminopenicilinas): por la hiperestimulación de linfocitos $\mathrm{T}$ que producen estas infecciones per se.

- Tipos de antígenos leucocitarios humanos (human leukocyte antigen; HLA, por sus siglas en inglés): hay pruebas de propensión familiar a desarrollar RHS graves a medicamentos concretos en presencia de ciertos tipos de alelos $H L A-B$. Por ejemplo, el síndrome de Stevens-Johnson (SSJ) y la necrólisis epidérmica tóxica (NET) por carbamazepina, en pacientes chinos de la etnia han, se asocian a la presencia de $H L A-B^{\star} 15025$; sin embargo,

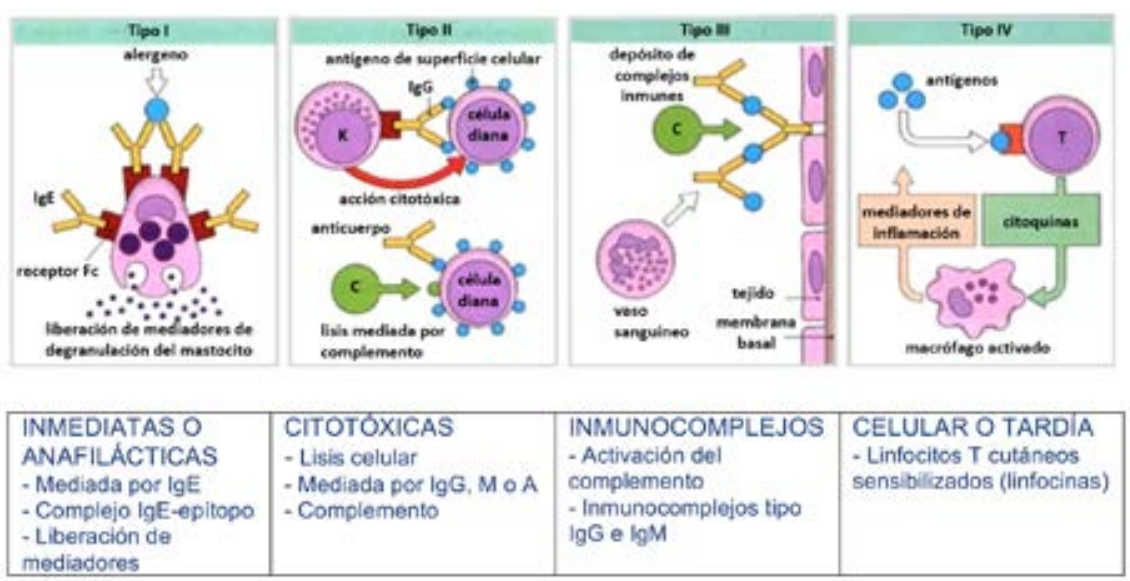

Tomado y adaptado de Brugaletta Matheus D. Hospital Universitario Virgen de la Arrixaca, Servicio de Alergología, 2009. Fc: fracción constante; IgG: inmunoglobulina G; IgE: inmunoglobulina E; IgM: inmunoglobulina M. 
en pacientes europeos, los $H L A-A * 3101$ tienen mayor riesgo de presentar estas RHS.

- El asma y las enfermedades atópicas en general no constituyen un factor de riesgo para padecerla, pero sí un agravante por la posibilidad de reacciones mediadas por inmunoglobulina $\mathrm{E}$ (IgE) de mayor magnitud clínica. ${ }^{4,6}$

- Antecedentes familiares de alergia a fármacos: los hijos de pacientes con alergia a fármacos tienen 10 veces mayor posibilidad de efectuar este tipo de reacciones con respecto a la población general. ${ }^{13}$

\section{FISIOPATOLOGÍA}

Los betalactámicos incluyen penicilinas, cefalosporinas, carbapenems, monobactámicos, cefems (moxalactam, cefoxitina) y ácido clavulánico. ${ }^{12}$ Estos comparten una estructura química común de cuatro carbonos denominada anillo betalactámico y, excepto los monobactámicos, pueden poseer un segundo anillo, que varía en cada grupo:

- Penicilinas: anillo tiazolidínico.

- Cefalosporinas: anillo dihidrotiazona.

- Carbapenems: anillo de 5 carbonos.

Todos poseen, al menos, una cadena lateral, que es la responsable de las diferencias dentro de cada grupo; cefalosporinas y carbapenems tienen dos cadenas laterales. Aunque no tengan la misma estructura nuclear, pueden tener cadenas laterales idénticas, lo que les otorga elevada reactividad alergénica (amoxicilina y cefadroxilo) $)^{14,20-23}$ (Tabla 2).

La clasificación clínica de RALB se basa en el intervalo de tiempo entre su administración y la aparición de síntomas: ${ }^{5}$

Inmediatas

Ocurren entre minutos y 6 horas luego de administrar el fármaco. Suelen estar mediadas por anticuerpos IgE específicos, responsables de RHS tipo I. ${ }^{5}$ Sus manifestaciones clínicas características varían desde reacciones leves, como urticaria, angioedema, conjuntivitis, rinitis, síntomas gastrointestinales (náuseas, vómitos, diarrea, dolor abdominal) y broncoespasmo, hasta graves, como shock anafiláctico. ${ }^{5,24}$

\section{Aceleradas}

Aparecen entre 1 y 72 horas tras la administración medicamentosa; su fisiopatogenia no está suficientemente aclarada; se manifiesta con urticaria, angioedema, edema laríngeo y sibilancias. ${ }^{5,25}$

Tardías

Tienen lugar a partir de las $72 \mathrm{~h}$ hasta varias semanas posteriores a la administración del fármaco. ${ }^{14,25}$

Algunas de estas RHS son mediadas por linfocitos $\mathrm{T}$ y sus manifestaciones clínicas asociadas son variadas: exantema medicamentoso fijo (EMF), dermatitis exfoliativa, exantema cutáneo con eosinofilia y síntomas sistémicos (drug rash with eosinophilia and systemic symptoms; $D R E S S$, por sus siglas en inglés), dermatitis exfoliativa, NET, SSJ, enfermedad del suero, reacciones de fotosensibilidad, exantema pustuloso generalizado agudo (acute generalized exanthematous pustulosis; $A G E P$, por sus siglas en inglés).

Fiebre, anemia, neutropenia, trombocitopenia, vasculitis, nefritis, artritis corresponden a RHS de tipo ii y iii, en las que los anticuerpos inmunoglobulina M (IgM) e inmunoglobulina G (IgG) y el complemento pasan a ser partícipes necesarios del desarrollo de estas reacciones. ${ }^{5,24}$

Las reacciones clínicas más comunes no suelen ser graves y consisten en exantemas y urticaria de difícil diagnóstico diferencial. Las reacciones aceleradas y tardías pueden agruparse como no inmediatas. ${ }^{5,24}$

Las RHS poseen dos fases: sensibilizante y efectora. La primera involucra la estimulación primaria y la expansión clonal de células T específicas para betalactámicos. ${ }^{25}$ Con su ingreso

TABLA 2. Fármacos betalactámicos que comparten cadenas laterales ${ }^{12}$

\begin{tabular}{lccc}
\hline Fármaco 1 & Fármaco 2 & Fármaco 3 & Fármaco 4 \\
\hline Amoxicilina & Cefadroxilo & Cefaclor & \\
Ampicilina & Cefalexina & & \\
Piperacilina & Cefoperazona & & \\
Aztreonam & Ceftazidima & Cefalexina & Cefaloridina \\
Bencilpenicilina & Cefalotina & & \\
Cefotaxima & Ceftriaxona & & \\
Cefuroxima & Cefoxitina & & \\
\hline
\end{tabular}


al organismo, las moléculas con características de hapteno, como las penicilinas, deben unirse a un carrier proteico para adquirir potencialidad antigénica. ${ }^{24}$ Si el complejo es soluble, es reconocido y presentado a linfocitos $\mathrm{B}$ para luego inducir la formación de IgE específica en RHS tipo I.

Otras moléculas hapténicas pueden también unirse a proteínas no solubles en la membrana celular. ${ }^{12}$ Estas pueden ser endocitadas y procesadas por otras células presentadoras de antígenos, como macrófagos, células dendríticas y de Langerhans, y luego ser reconocidas por linfocitos $\mathrm{B}$ o linfocitos $\mathrm{T}$ con apropiada especificidad para producir anticuerpos $\operatorname{IgM~o}$ IgG o activación de células T.24,25

Una hipótesis alternativa sería el llamado concepto $\mathrm{p}-\mathrm{i},{ }^{26,27}$ que propone la existencia de interacciones directas (sin procesamiento intracelular previo) de la droga con el receptor inmune (receptores de célula $\mathrm{T}$ o moléculas de HLA). Esta interacción podría modificar la hendidura de unión a péptido de la molécula de HLA y producir activación de células $\mathrm{T}$ específicas. ${ }^{27,28}$

Luego de la sensibilización, una reexposición al medicamento causa que las células $\mathrm{T}$ afectadas o los anticuerpos específicos entren en fase efectora, lo que genera RHS tipo I, II, III o IV, responsables de las manifestaciones clínicas ${ }^{24}$ (Tabla 3).

La mayoría de las RALB son tipo I o IV. ${ }^{5,24}$ Los mecanismos implicados en la patogenia de las RALB tienen su correlato clínico específico (Tabla 3).

\section{CLÍNICA}

Las manifestaciones clínicas son variadas, predominantemente, cutáneas, y pueden asociarse al mecanismo fisiopatológico productor, de forma tal que una historia clínica detallada y un examen físico pertinaz aportarán importantes datos para el diagnóstico y orientarán a la elección de la metodología de estudio específica. Estos datos, a modo de resumen práctico para el consultorio del pediatra, se detallan en la Tabla 4.

\section{METODOLOGÍA DIAGNÓSTICA}

Es elemental confeccionar una detallada historia clínica, determinando el tiempo entre la exposición y el inicio de la reacción, las vías de administración, evaluar la patología de base y la utilización conjunta de otros fármacos. Se debe investigar la tolerancia previa y posterior a los

TABLA 3. Mecanismos implicados en la patogenia de las reacciones alérgicas a betalactámicos y su correlato clínico ${ }^{3,4}$

\begin{tabular}{|c|c|c|c|}
\hline $\begin{array}{l}\text { Tipo de respuesta } \\
\text { inmune }\end{array}$ & $\begin{array}{c}\text { Fisiopatología de la reacción } \\
\text { (después de la administración } \\
\text { del fármaco) } \\
\end{array}$ & Cronología & Síntomas clínicos \\
\hline I. IgE & $\begin{array}{l}\text { Degranulación de mastocitos } \\
\text { y basófilos }\end{array}$ & Entre minutos y $6 \mathrm{~h}$ & $\begin{array}{l}\text { Shock anafiláctico, } \\
\text { angioedema, urticaria, } \\
\text { broncoespasmo }\end{array}$ \\
\hline II. IgG y complemento & $\begin{array}{l}\text { IgG y citotoxicidad dependiente } \\
\text { de complemento }\end{array}$ & Entre 5 y 15 días & Citopenias \\
\hline III. IgM o IgG y complemento & Depósito de inmunocomplejos & $\begin{array}{l}\text { De } 7 \text { a } 8 \text { días en la } \\
\text { enfermedad del suero/ } \\
\text { urticaria; de } 7 \text { a } 21 \text { días } \\
\text { en la vasculitis }\end{array}$ & $\begin{array}{l}\text { Enfermedad del suero, } \\
\text { urticaria, vasculitis }\end{array}$ \\
\hline IV. a. Th1 (IFN $\gamma)$ & $\begin{array}{l}\text { Reclutamiento y } \\
\text { activación de monocitos }\end{array}$ & De 1 a 21 días & Eccema \\
\hline IV. b. Th2 (IL-4 e IL-5) & $\begin{array}{l}\text { Reclutamiento y } \\
\text { activación de eosinófilos }\end{array}$ & $\begin{array}{l}\text { De1 a varios días } \\
\text { tras iniciar el fármaco }\end{array}$ & $\begin{array}{l}\text { Exantema } \\
\text { maculopapular, DRESS }\end{array}$ \\
\hline $\begin{array}{l}\text { IV. c. Células T citotóxicas } \\
\text { (perforina, granzima B, FasL) }\end{array}$ & $\begin{array}{l}\text { Acción citotóxica de linfocitos } \\
\text { CD } 4+\text { y CD } 8+\text { produce aceleración } \\
\text { de apoptosis de queratinocitos }\end{array}$ & $\begin{array}{l}\text { De 1-2 días. } \\
\text { En EMF, 4-28 días }\end{array}$ & $\begin{array}{l}\text { Exantema maculopapular, } \\
\text { EMF, SSJ, NET, } \\
\text { exantema bulloso }\end{array}$ \\
\hline IV. d. Células T (IL-8/CXCL8) & $\begin{array}{l}\text { Reclutamiento y } \\
\text { activación de neutrófilos }\end{array}$ & De 1 a 2 días o más & AGEP \\
\hline
\end{tabular}

CD: cluster of differentiation (grupo o cúmulo de diferenciación; CXCL8: interleukina 8; DRESS: exantema cutáneo con eosinofilia y síntomas sistémicos; EMF: exantema medicamentoso fijo; FasL: proteína de membrana miembro de la familia del factor de necrosis tumoral; h: horas; IgE: inmunoglobulina E; IgG: inmunoglobulina G; IFN $\gamma$ : interferón gama; IL: interleukina; NET: necrólisis epidérmica tóxica; PPC: prueba de provocación controlada; SSJ: síndrome de Stevens-Johnson; Th: linfocitos T helper; +: positivo. 
TABla 4. Mecanismos fisiopatológicos y manifestaciones clínicas de reacciones alérgicas a betalactámicos

\begin{tabular}{|c|c|c|c|c|}
\hline \multicolumn{2}{|c|}{ Mecanismo/RHS } & Patologías & $\begin{array}{l}\text { Características clínicas/ } \\
\text { Puntos claves }\end{array}$ & $\begin{array}{l}\text { Diagnósticos } \\
\text { direrenciales }\end{array}$ \\
\hline \multicolumn{2}{|l|}{$\begin{array}{l}\text { Inmediato } \\
\text { RHS tipo I } \\
\text { IgE mediada }\end{array}$} & Urticaria & $\begin{array}{l}\text { La más frecuente. }{ }^{10} \text { Exantema maculopapular } \\
\text { eritematoso, con o sin angioedema. }\end{array}$ & $\begin{array}{l}\text { Otros fármacos: AINE, opioides, } \\
\text { sulfamidas }{ }^{29}\end{array}$ \\
\hline \multirow{4}{*}{\multicolumn{2}{|c|}{$\begin{array}{l}\text { De minutos a } 6 \text { h, } \\
\text { generalmente, en } \\
\text { la } 1 .^{a} \text { hora de la } \\
\text { administración } \\
\text { del antibiótico, } \\
\text { independientemente } \\
\text { de la VA }{ }^{13,29}\end{array}$}} & Angioedema & $\begin{array}{l}\text { Aislado es raro, generalmente, } \\
\text { asociado a urticaria. }{ }^{10}\end{array}$ & $\begin{array}{l}\text { Otros fármacos: } \\
\text { AINE, IECA, sulfamidas }{ }^{29}\end{array}$ \\
\hline & & $\begin{array}{l}\text { Broncoespasmo } \\
\text { Laringotraqueítis } \\
\text { Conjuntivitis }\end{array}$ & $\begin{array}{l}\text { Afectación de la vía aérea superior } \\
\text { con o sin síntomas bronquiales, } \\
\text { disnea inspiratoria, disfonía, } \\
\text { sialorrea, edema laríngeo. }{ }^{5}\end{array}$ & $\begin{array}{l}\text { Otros fármacos: AINE, } \\
\text { sulfamidas }{ }^{29}\end{array}$ \\
\hline & & Anafilaxia & Síntomas sistémicos. ${ }^{5}$ & Otros fármacos: AINE, sulfamidas ${ }^{29}$ \\
\hline & & $\begin{array}{l}\text { Síntomas } \\
\text { gastrointestinales }\end{array}$ & $\begin{array}{l}\text { Náuseas, vómitos, } \\
\text { dolor abdominal, diarrea. }\end{array}$ & $\begin{array}{l}\text { Otros fármacos: AINE, } \\
\text { sulfamidas }{ }^{29}\end{array}$ \\
\hline \multicolumn{2}{|l|}{$\begin{array}{l}\text { Tardías } \\
\text { RHS tipo II } \\
\text { Citotóxicas }\end{array}$} & $\begin{array}{l}\text { Anemia } \\
\text { hemolítica }^{13}\end{array}$ & $\begin{array}{l}\text { Infrecuentes. En terapias prolongadas } \\
\text { con dosis altas de penicilina. } \\
\text { Cefaleas, fatiga, decaimiento, } \\
\text { problemas de concentración, } \\
\text { mareos, palidez, esplenomegalia. }\end{array}$ & $\begin{array}{l}\text { Exposición a otros fármacos, } \\
\text { químicos y toxinas }\end{array}$ \\
\hline \multirow{2}{*}{\multicolumn{2}{|c|}{$\begin{array}{l}\text { Comienzo entre } \\
5 \text { y } 15 \text { días luego de } \\
\text { la administración } \\
\text { del antibiótico }^{13}\end{array}$}} & Trombocitopenia $^{13}$ & $\begin{array}{l}\text { Hematomas espontáneos, lesiones purpúricas, } \\
\text { epistaxis, gingivorragias, hematemesis, mareos, } \\
\text { debilidad, hemorragias digestivas. }\end{array}$ & Otros fármacos: sulfamidas \\
\hline & & Leucopenia $^{13}$ & Linfopenia y/o neutropenia & $\begin{array}{l}\text { Otros fármacos: sulfamidas, } \\
\text { citopenias de otro origen }\end{array}$ \\
\hline \multicolumn{2}{|c|}{$\begin{array}{l}\text { Tardias } \\
\text { RHS tipo III } \\
\text { Por inmunocomplejos }\end{array}$} & $\begin{array}{l}\text { Enfermedad del } \\
\text { suero }\end{array}$ & $\begin{array}{l}\text { Rara. Fiebre, exantema maculopapular, } \\
\text { pruriginoso y simétrico, linfadenopatía, } \\
\text { afectación renal y/o hepática, artralgias. }{ }^{30,31}\end{array}$ & Otros fármacos: cefaclor ${ }^{5}$ \\
\hline \multicolumn{2}{|c|}{$\begin{array}{l}\text { Manifestaciones en } \\
\text { 3-4 semanas del uso } \\
\text { del fármaco }{ }^{13}\end{array}$} & Vasculitis & $\begin{array}{l}\text { El } 10 \% \text {, etiología medicamentosa: vasculitis } \\
\text { leucocitoclásticas (púrpura, petequias y } \\
\text { equimosis en los miembros inferiores simétricas) }\end{array}$ & Otros fármacos: sulfamidas \\
\hline \multirow[t]{3}{*}{$\begin{array}{l}\text { Tardias } \\
\text { RHS tipo IV } \\
\text { Mediadas por } \\
\text { linfocitos } \mathrm{T}^{13}\end{array}$} & IV. a. & $\begin{array}{l}\text { Eccema o } \\
\text { dermatitis } \\
\text { de contacto }\end{array}$ & $\begin{array}{l}\text { Trabajadores sanitarios o de la industria } \\
\text { manufacturera. Provocado por el uso tópico } \\
\text { de penicilina. }{ }^{13} \text { Aparece } 1-21 \text { días } \\
\text { del inicio del tratamiento. }{ }^{33}\end{array}$ & \\
\hline & IV. b. & $\begin{array}{l}\text { Exantema } \\
\text { maculopapular } \\
\text { Exantema } \\
\text { morbiliforme }\end{array}$ & $\begin{array}{l}\text { Inicia 3-4 días después del uso o } 1-2 \mathrm{~h} \text { desde } \\
\text { la última administración. }{ }^{13} \text { Aparece en el } 10 \% \\
\text { de los pacientes con ampicilina y amoxicilina } \\
\text { y en el } 70 \% \text { con VEB que utilizan aminopenicilir }\end{array}$ & Sulfamidas, anticonvulsivantes \\
\hline & & DRESS & $\begin{array}{l}\text { Rara y grave en niños. }{ }^{34} \text { Frecuente en VIH o } \\
\text { citomegalovirus. }{ }^{13} \text { Aparece } 2-6 \text { semanas } \\
\text { después del inicio. }{ }^{33} \\
\text { Síntomas: fatiga, prurito, fiebre, rash } \\
\text { maculopapular morbiliforme diseminado. } \\
\text { Diagnóstico. Más del } 50 \% \text { de la superficie } \\
\text { corporal afectada y/o dos de los siguientes } \\
\text { signos: edema facial, lesiones infiltradas, } \\
\text { escamosas, púrpuras, adenomegalias (el 30-60 \%) } \\
\text { linfocitos atípicos, eosinofilia, } \\
\text { disfunción multiorgánica. }{ }^{35}\end{array}$ & $\begin{array}{l}\text { Otros fármacos: tetraciclinas } \\
\text { sulfamidas, anticonvulsivantes, } \\
\text { y/o antirretrovirales. }{ }^{35}\end{array}$ \\
\hline
\end{tabular}


TABLA 4. (Continuación)

\begin{tabular}{|c|c|c|c|}
\hline IV. c. & SSJ & \multicolumn{2}{|c|}{$\begin{array}{l}\text { (desprendimiento cutáneo menor del } 10 \% \text { : SSJ; } \\
\text { mayor del } 30 \% \text { : NET) }{ }^{35} \text { prurito ocular y disfagia, } \\
\text { eritema, erosiones, úlceras, costras y } \\
\text { pseudomembranas en la mucosa oral, } \\
\text { ocular y genital. }^{33}\end{array}$} \\
\hline & NET & \multicolumn{2}{|c|}{$\begin{array}{l}\text { Fiebre, malestar general, lesiones de piel y mucosas, } \\
\text { anemia, linfopenia. } \\
\text { Graves: hipoalbuminemia, alteraciones electrolíticas, } \\
\text { hiperglucemia, urea elevada. }^{35}\end{array}$} \\
\hline & EMF & $\begin{array}{l}\text { Mácula fija luego de } 30 \text { minutos- } 16 \text { h de } \\
\text { administrar el medicamento; evoluciona a } \\
\text { edema y dolor. Mejora en días o semanas y } \\
\text { deja una mácula más oscura. }{ }^{35}\end{array}$ & $\begin{array}{l}\text { Otros fármacos: TMS, AINE, } \\
\text { tetraciclinas, pseudoefedrina. }{ }^{29,35}\end{array}$ \\
\hline IV. d. & AGEP & $\begin{array}{l}\text { Rara en niños, relacionada con infecciones. } \\
\text { Comienzo agudo, } 1-2 \text { días después de iniciar } \\
\text { el tratamiento. }{ }^{33} \text { Eritema asintomático o } \\
\text { levemente pruriginoso, pústulas foliculares } \\
\text { de menos de } 5 \text { mm de diámetro, con fiebre }{ }^{29} \\
\text { y mal estado general. }{ }^{35} \\
\text { No es necesaria la sensibilidad previa. }{ }^{35}\end{array}$ & $\begin{array}{l}\text { Otros fármacos: cefixima, } \\
\text { vancomicina, macrólidos, } \\
\text { paracetamol. } .^{29,35}\end{array}$ \\
\hline
\end{tabular}

AGEP: exantema pustuloso generalizado agudo; AINE: antiinflamatorios no esteroideos; DRESS: exantema cutáneo con eosinofilia y síntomas sistémicos; EMF: exantema medicamentoso fijo; IECA: inhibidores de la enzima convertidora de angiotensina; IgE: inmunoglobulina E; NET: necrólisis epidérmica tóxica; $1 .^{a}$ : primera hora; \%: por ciento; RHS: reacciones de hipersensibilidad; SSJ: síndrome de Stevens-Johnson; TMS: trimetoprima sulfametoxazol; VA: vía de administración; VEB: virus de Epstein-Barr; VIH: virus de la inmunodeficiencia adquirida humana.

fármacos del grupo y a alternativos (macrólidos y sulfonamidas). Es esencial estudiarlas para diferenciar las RALB de aquellas producidas por combinación de virus y antibiótico. ${ }^{14}$

Si la reacción ocurrió años antes, la información puede ser inexacta y/o el paciente pudo haber perdido la sensibilización; esto obliga a profundizar algoritmos diagnósticos con métodos específicos. ${ }^{13}$

Con respecto a los métodos in vitro, la IgE sérica específica es especialmente útil ante reacciones inmediatas, recientes, cuando los test cutáneos son negativos o cuando no es posible realizarlos por enfermedad cutánea difusa o imposibilidad de suspender medicamentos que afectan el resultado del test cutáneo. Una desventaja de estos estudios es que el paso del tiempo produce depleción de los anticuerpos, y se han reportado falsos negativos cuando la prueba se realizó cerca de la reacción aguda (antes de las 4 semanas) o cuando se retrasó más allá de 6 meses. $^{14}$
El método in vitro fluoroenzimoinmunoensayo detecta IgE sérica específica para alérgenos. Tiene la ventaja sobre métodos más antiguos, como el radio allergo sorbent test $(R A S T)$ y el radioinmunoanálisis de no usar isótopos radioactivos. El fluoroenzimoinmunoensayo es una de las técnicas más validadas y considerada estándar de referencia (resultados más sensibles) en métodos in vitro para la detección de IgE sérica específica. . $^{13,35,36}$

El test de InmunoCAP ${ }^{\circledR}$ usa este método en fase sólida de polímero de celulosa. La validación no alcanza a todos los alérgenos; su valor de corte considerado positivo es $0,35 \mathrm{Ku} / \mathrm{L}^{36}$

En Argentina, por su disponibilidad, prevalece el uso del ensayo por inmunoabsorción ligado a enzimas (enzyme-linked immunosorbent assay; ELISA, por sus siglas en inglés) o el RAST para determinar IgE específica a betalactámicos (bencilpenicilina y fenoximetilpenicilina, amoxicilina, ampicilina y cefalosporinas).

Otras pruebas in vitro son el test de activación 
de basófilos $(\mathrm{TAB})$ y el test de transformación linfocitaria (TTL), ya que ciertas drogas, como penicilina $G$, pueden estimular la proliferación linfocitaria. ${ }^{36}$ El TTL es considerado método experimental, ${ }^{37}$ aunque puede ser de utilidad en RHS no inmediatas. El TAB, con la expresión de CD63 y CD203c, puede colaborar en el diagnóstico de expresiones graves, ${ }^{38,39}$ siempre que se realice dentro de los primeros doce meses, ya que tiende a negativizarse con el tiempo. ${ }^{40}$ También puede ser de utilidad en el diagnóstico de RHS con ácido clavulánico asociado a amoxicilina, con alto valor predictivo positivo. ${ }^{41,42}$

Los métodos in vivo son las pruebas cutáneas de lectura inmediata (PCLI), pruebas intradérmicas (PI) inmediatas o retardadas, test del parche atópico (TPA) y pruebas de provocación controlada (PPC), como el test de provocación oral (TPO).

Con respecto a las PCLI, tienen utilidad en las RHS tipo I, mediadas por IgE, cuando resultan positivas, $\mathrm{y}$, por descarte, en las RHS tipo iv, mediadas por linfocitos $\mathrm{T}$ específicos, cuando resultan negativas. ${ }^{14}$ Deben realizarlas especialistas en Alergia, ya que se requiere experiencia para interpretar los resultados y tratar cualquier complicación, y luego de 4-8 semanas posteriores a la reacción, debido a la depleción de mediadores producidos en las células efectoras. ${ }^{14}$

La sensibilidad de PCLI disminuye con el tiempo por descenso paulatino de niveles de IgE específica en pacientes no reexpuestos, más rápidamente en aminopenicilinas que penicilina. ${ }^{44}$ Para efectuarlas, los pacientes no deben recibir antihistamínicos desde 10 días previos a la prueba, y pueden utilizarse las presentaciones orales y parenterales del fármaco, así como determinantes mayores (PPL), que representan el anillo betalactámico, común a todo el grupo (el $95 \%)$, y los determinantes menores (minor determinants; $M D M$, por sus siglas en inglés), el $5 \%$ restante. ${ }^{14}$

En Argentina, no se comercializan PPL, por lo cual la penicilina $\mathrm{G}$, un importante $M D M$, es útil para el diagnóstico.

Como el valor predictivo negativo de la PCLI es bajo, si resulta negativa, requiere confirmar el diagnóstico con TPO o, en casos seleccionados, PI de lectura inmediata con la evaluación previa de riesgo. ${ }^{13}$

La PI de lectura retardada y/o TPA se utilizan en las RHS tipo IV, aunque sus resultados no son consistentes (valor predictivo incierto). ${ }^{44} \mathrm{El} \mathrm{TPA}$ es seguro en reacciones cutáneas graves, como

FIGURA 2. Esquema protocolo de estudio en reacciones alérgicas a betalactámicos

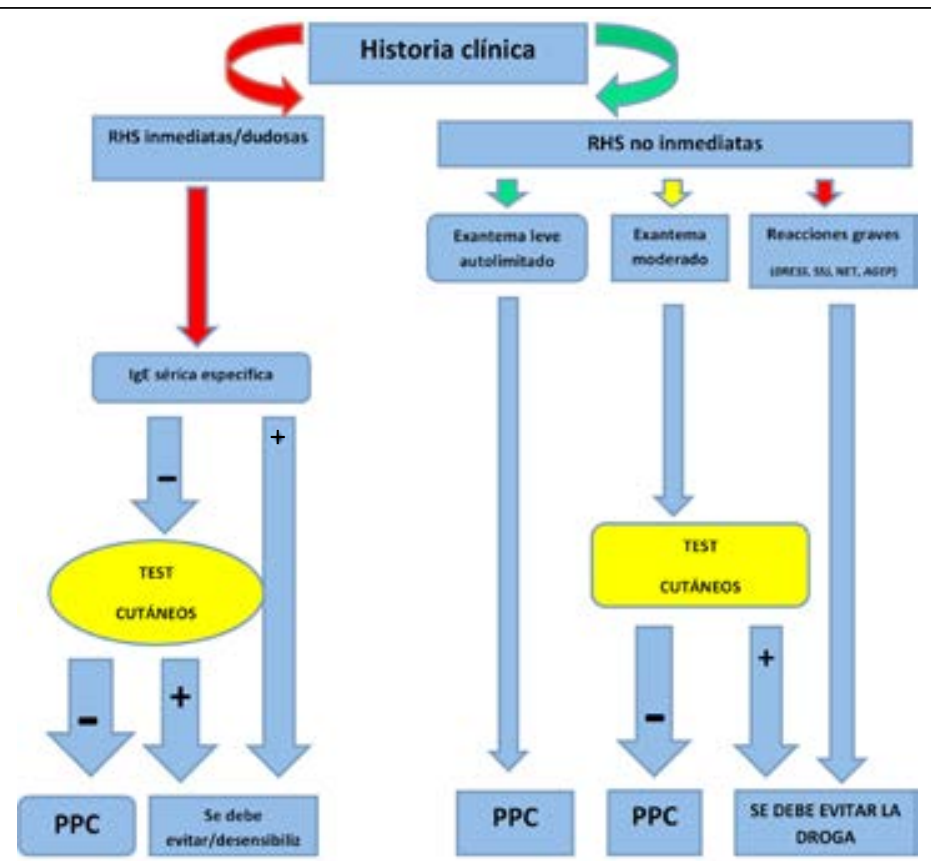

IgE: inmunoglobulina E; RHS: reacciones de hipersensibilidad; PPC: prueba de provocación controlada; DRESS: exantema cutáneo con eosinofilia y síntomas sistémicos; SSJ: síndrome de Stevens-Johnson;

NET: necrólisis epidérmica tóxica; AGEP: exantema pustuloso generalizado agudo; +: positivo; -: negativo. 
SSJ, NET, DRESS y AGEP. ${ }^{13}$ Aproximadamente, $1 / 3$ de pacientes alérgicos a penicilina tienen PCLI y TPA negativos; solo se diagnostican por TPO. ${ }^{13}$

En las PPC, y, de manera específica, en el TPO, el alergólogo entrenado, con la firma previa del consentimiento informado, expone al paciente al fármaco sospechoso, administrando dosis gradualmente crecientes hasta llegar a dosis terapéuticas. ${ }^{14}$ Las primeras dosis deben realizarse en un ambiente controlado; luego, puede continuar en forma ambulatoria por 5-7 días, evaluando las RHS inmediatas y tardías. No deben efectuarse con antecedente de reacción anafiláctica por el uso del fármaco ni en SSJ, NET, AGEP, DRESS o en asma inestable. ${ }^{44,45,46}$ El médico especialista determinará si el TPO es una alternativa útil, ya que debe realizarse en pacientes con bajo riesgo de ser alérgicos al antibiótico ${ }^{47}$ o para excluir la reactividad cruzada. ${ }^{44}$ También explicará a los padres las características del test, los riesgos y los beneficios (Figura 2). De acuerdo con el tiempo transcurrido entre la reacción y la evaluación de los síntomas, ${ }^{45}$ algunos pacientes requerirán una nueva provocación 15 días después de la primera. ${ }^{14}$

Las RHS tipo II se estudian con alguna de las siguientes determinaciones: hemograma, para detectar la disminución de células hemáticas, la prueba de Coombs directa, la prueba indirecta de la antiglobulina, la microscopía fluorescente, las pruebas antirreceptor. ${ }^{48,49}$

En las RHS tipo III, las pruebas diagnósticas se basan en la capacidad de los inmunocomplejos, generados por la unión del medicamento o su metabolito con el anticuerpo, de reaccionar con el complemento, para lo que se utiliza el test de unión al Ciq. En interacción de inmunocomplejos que en su interior contengan componentes del complemento, con los receptores celulares, se utiliza el test de células de Raji. ${ }^{48-50}$

\section{TRATAMIENTO}

Se basa en la identificación del cuadro clínico, la droga responsable y el manejo de los síntomas. ${ }^{51}$ Ante la sospecha de RALB, la primera medida es suspenderlo.

Si la reacción es urticaria, con o sin angioedema y sin compromiso sistémico, puede ser suficiente el uso de antihistamínicos de primera o segunda generación. ${ }^{6}$ Si existe broncoespasmo, se requerirá el uso de broncodilatadores, oxigenoterapia, medidas de reanimación y/o soporte vital dependiendo de la gravedad. ${ }^{6}$

La mayoría de las RALB no presenta anafilaxia. Si impresiona progresión hacia shock anafiláctico, se recomienda la administración precoz de adrenalina intramuscular, con hospitalización y control por 24 horas (anafilaxia bifásica). ${ }^{6}$

En urticarias tardías y exantemas maculopapulares leves, se indican antihistamínicos, y puede utilizarse un tratamiento corto con prednisona si la reacción es más grave (1-2 mg/k/ día). ${ }^{6}$

Las RHS graves, como SSJ, NET, DRESS y $A G E P$, pueden requerir ingresar a la Unidad de Terapia Intensiva Pediátrica (UTIP) por su alto índice de mortalidad. ${ }^{52,53}$

Si se requiere un tratamiento farmacológico alternativo, se debe considerar que posea efectos terapéuticos similares, posibles reacciones cruzadas y equivalencia en su estructura química. Los antibióticos alternativos más utilizados son macrólidos (eritromicina, azitromicina y claritromicina), clindamicina, trimetoprimasulfametoxazol y gentamicina, según la

TABLA 5. Indicaciones y contraindicaciones de desensibilización ${ }^{55,56}$

\begin{tabular}{ll}
\hline \multicolumn{1}{c}{ Indicaciones } & \multicolumn{1}{c}{ Contraindicaciones } \\
\hline $\begin{array}{l}\text { Sífilis en el embarazo, sífilis congénita y neurosífilis. } \\
\text { Fibrosis quística }\end{array}$ & Asma no controlada (FEV1 $<70 \%)$. \\
$\begin{array}{l}\text { Infecciones por microorganismos multirresistentes susceptibles } \\
\text { solo a penicilina (endocarditis bacteriana subaguda } \\
\text { por enterococo, listeriosis, septicemia por estafilococo). }\end{array}$ & $\begin{array}{l}\text { Pacientes hemodinámicamente inestables. } \\
\text { Enfermedades cardíacas no controladas. } \\
\text { Uso de betabloqueantes. }\end{array}$ \\
& Anafilaxia grave. \\
& Insuficiencia hepática o renal \\
\hline
\end{tabular}

<: menor; NET: necrólisis epidérmica tóxica; \%: por ciento; SSJ: síndrome de Stevens-Johnson;

DRESS: exantema cutáneo con eosinofilia y síntomas sistémicos; VEF1: volumen espiratorio forzado en 1 segundo. 
infección para tratar y la sensibilidad estudiada por antibiograma de la bacteria implicada. En las infecciones graves, pueden utilizarse aztreonam (monobactámico con reactividad cruzada excepcional) y /o vancomicina. ${ }^{54} \mathrm{En}$ las infecciones respiratorias graves, pueden emplearse quinolonas (levofloxacina), con el consentimiento informado previo de los padres. Ante la falta de alternativa terapéutica, la desensibilización estaría indicada. Sin embargo, existen situaciones en las que dicha práctica se contraindica (Tabla 5).

La desensibilización es un procedimiento por el cual al paciente con diagnóstico confirmado de RALB se le administra, en dosis crecientes, el fármaco implicado, con el propósito de inhibir temporalmente su capacidad de respuesta alérgica, lo que induce la tolerancia inmunológica. ${ }^{5,951}$ Se pueden utilizar por vía oral y parenteral, y ambas son igualmente efectivas. La vía oral es más segura, fácil y económica. Sin embargo, no siempre es aconsejable o factible. ${ }^{52,53}$

La desensibilización a drogas es un procedimiento que modifica la respuesta inmune para promover la tolerancia en lugar de hipersensibilidad; debe realizarse cuando el paciente tiene alergia al fármaco, requiere utilizarlo y no existen alternativas. ${ }^{10} \mathrm{La}$ administración repetida de dosis subumbrales del medicamento proporciona suficientes determinantes antigénicos para unir IgE en superficie de basófilos y mastocitos sin enlaces cruzados, lo que hace que estas células no respondan a una mayor dosis. ${ }^{54,55}$

Aproximadamente, $1 / 3$ de pacientes desarrollará una RHS durante el procedimiento, que tiende a ser leve. Esto no contraindica la continuación del procedimiento, sino que la siguiente dosis para aplicar será 10 veces menor. La tasa de efectividad se ha estimado entre el $58 \%$ y el $100 \% .^{13}$

Una desensibilización eficaz se puede observar con disminución del tamaño o negativización de la PCLI del fármaco implicado. Esto confirma la abolición temporal de la respuesta alérgica. Se considera que la desensibilización pierde su efectividad al suspender la administración del fármaco por un período superior a 48 horas.

Es un procedimiento de riesgo; debe ser controlado por un alergólogo, con consentimiento informado, en el marco de la UTIP. Existen diversos protocolos ${ }^{51,56-59}$ (Tabla 6 y 7 ). Para desensibilizar, también pueden utilizarse otros derivados betalactámicos, como el imipenem y el meropenem. ${ }^{51}$

En las RALB, se debe evitar la droga y su familia de fármacos, educar al paciente y/o cuidadores en tal sentido, así como en el reconocimiento temprano y el tratamiento de reacciones graves, como anafilaxia. ${ }^{10}$ Es aconsejable identificar al paciente en la historia clínica y solicitar a la familia que el niño cuente con algún elemento identificatorio, como tarjetas, pulseras o medallas. ${ }^{10}$

Tabla 6. Protocolo de desensibilización con penicilina oral

\begin{tabular}{|c|c|c|c|c|}
\hline $\begin{array}{l}\text { Pasos } \\
\text { Dosis } \\
\text { N. }{ }^{*} \\
\end{array}$ & $\begin{array}{c}\text { Dosis } \\
\text { Concentración } \\
(\mathrm{mg} / \mathrm{ml})\end{array}$ & $\begin{array}{c}\text { Volumen/paso } \\
\text { (ml en } 30 \mathrm{ml} \\
\text { de agua o jarabe) }\end{array}$ & $\begin{array}{c}\text { Dosis/paso } \\
\text { acumulada } \\
\text { (mg/unidades) }\end{array}$ & $\begin{array}{c}\text { Dosis } \\
\text { (mg/unidades) }\end{array}$ \\
\hline 1 & 0,5 & 0,10 & $0,05 / 100$ & $0,05 / 100$ \\
\hline 2 & & 0,20 & $0,10 / 200$ & $0,15 / 300$ \\
\hline 3 & & 0,40 & $0,20 / 400$ & $0,35 / 700$ \\
\hline 4 & & 0,80 & $0,40 / 800$ & $0,75 / 1500$ \\
\hline 5 & & 1,60 & $0,80 / 1600$ & $1,55 / 3100$ \\
\hline 6 & & 3,20 & $1,60 / 3200$ & $3,15 / 6300$ \\
\hline 7 & & 6,40 & $3,20 / 6400$ & $6,35 / 12700$ \\
\hline 8 & 5 & 1,20 & $6 / 12000$ & $12,35 / 24700$ \\
\hline 9 & & 2,40 & $12 / 24000$ & $24,35 / 48700$ \\
\hline 10 & & 4,80 & $24 / 48000$ & $48,35 / 96700$ \\
\hline 11 & 50 & 1 & $50 / 80000$ & $98,35 / 176700$ \\
\hline 12 & & 2 & $100 / 160000$ & $198,35 / 336700$ \\
\hline 13 & & 4 & $200 / 320000$ & $398,35 / 656700$ \\
\hline 14 & & 8 & $400 / 640000$ & $798,35 / 1296700$ \\
\hline
\end{tabular}

* Intervalo entre dosis: 15 minutos.

N. ${ }^{\circ}$ : número; ml: mililitros; mg: miligramos; /: por.

Tomado y Adaptado de De las Vecillas Sánchez L y col. ${ }^{59}$ 


\section{MEDIDAS PREVENTIVAS}

No hay evidencia concluyente para predecir qué pacientes desarrollarán RALB y cómo prevenirla. Sin embargo, algunos factores deben ser considerados:

- Utilizar betalactámicos solo cuando estén indicados, y evitar su uso en procesos febriles $\sin$ foco, reducirá la incidencia de alergia. ${ }^{20}$

- La penicilina tópica favorece la sensibilización alérgica, por lo cual debe evitarse. ${ }^{13}$

- En relación con la genética y la historia familiar, la evidencia disponible no permite establecer una asociación significativa como predisponente; no obstante, parece tener más relevancia en las RHS tardías. ${ }^{13}$

- Tener precaución en pacientes que requieren antibióticos frecuentemente (enfermedad fibroquística)..$^{13}$

- La correcta identificación de las RALB es primordial para la prevención de futuras reacciones. ${ }^{19}$

- Una rápida intervención diagnóstica evitará prolongar el rótulo de alérgico en la edad adulta y el uso innecesario de antibióticos alternativos, frecuentemente, más caros y menos efectivos, tendientes a generar multirresistencia. ${ }^{57-59}$

\section{CONCLUSIONES}

Las RALB son un importante problema en la infancia, debido a que son los antibióticos más usados y los más accesibles a través de programas sociales o para el costo de bolsillo de las familias. Existen nuevos métodos diagnósticos que no se hallan disponibles todavía en nuestro país. Sin embargo, desde la especialidad de Alergia e Inmunología Infantil, se cuenta con métodos validados para realizar un diagnóstico pertinente. El diagnóstico de certeza en las RALB es primordial para evitar riesgos en los niños y mejorar los costos en los sistemas de salud.

\section{REFERENCIAS}

1. Cortada Macías JM, López Serrano MC, Blasco Sarramián A, Mayorga C, et al. Introducción, conceptos generales, epidemiología. Fisiopatología: los fármacos como antígenos. En: Peláez Hernández A, Dávila González IJ. Tratado de Alergología. Madrid: Ergón; 2007.Págs.1297-324.

2. Vervloet D, Durham S. Adverse reactions to drugs. BMJ. 1998; 316(7143):1511-4.

3. Gell P, Combs R (eds.). Clinical aspects of immunology. Oxford: Blackwell Science; 1964.

4. González Cervera J. Alergia a medicamentos en Atención Primaria. Aproximación práctica. VI Foro de Pediatría de Atención Primaria de Extremadura. 2009.Págs.27-35. [Consulta: 29 de octubre de 2018]. Disponible en: https: / / spapex.es / foro-pediatrico/2009/alergia-medicamentos.

5. Demoly P, Adkinson NF, Brockow K, Castells M, et al.

Tabla 7. Protocolo de desensibilización con penicilina parenteral

\begin{tabular}{|c|c|c|c|c|c|}
\hline $\begin{array}{l}\text { Pasos } \\
\text { (dosis) } \\
\text { N. }^{\circ} \\
\end{array}$ & $\begin{array}{c}\text { Concentración de } \\
\text { betalactámicos } \\
(\mathrm{mg} / \mathrm{ml})\end{array}$ & $\begin{array}{c}\text { Concentración de } \\
\text { penicilina } G \\
(\mathrm{U} / \mathrm{ml})\end{array}$ & $\begin{array}{c}\text { Volumen } \\
(\mathrm{ml})\end{array}$ & $\begin{array}{c}\text { Dosis/unidades } \\
(\mathrm{mg} / \mathrm{U})\end{array}$ & $\begin{array}{c}\text { Dosis } \\
\text { acumulada }(\mathrm{U})\end{array}$ \\
\hline 1 & 0,1 & 160 & 0,10 & $0,01 / 16$ & 16 \\
\hline 2 & & & 0,20 & $0,02 / 32$ & 48 \\
\hline 3 & & & 0,40 & $0,04 / 64$ & 112 \\
\hline 4 & & & 0,80 & $0,08 / 128$ & 140 \\
\hline 5 & 1,0 & 1600 & 0,15 & $0,15 / 240$ & 380 \\
\hline 6 & & & 0,30 & $0,30 / 480$ & 860 \\
\hline 7 & & & 0,60 & $0,60 / 960$ & 1820 \\
\hline 8 & & & 1,00 & $1,00 / 1600$ & 3420 \\
\hline 9 & 10,0 & 16000 & 0,20 & $2,00 / 3200$ & 6620 \\
\hline 10 & & & 0,40 & $4,00 / 6400$ & 13020 \\
\hline 11 & & & 0,80 & $8,00 / 12800$ & 25820 \\
\hline 12 & 100,0 & 160000 & 0,15 & $15 / 24000$ & 49820 \\
\hline 13 & & & 0,30 & $30 / 48000$ & 98820 \\
\hline 14 & & & 0,60 & $60 / 96000$ & 194820 \\
\hline 15 & & & 1,00 & $100 / 160000$ & 354820 \\
\hline 16 & 1000 & 1600000 & 0,20 & $200 / 320000$ & 674820 \\
\hline 17 & & & 0,40 & $400 / 640000$ & 1304820 \\
\hline 18 & & & 0,80 & $800 / 1280000$ & 2584000 \\
\hline
\end{tabular}

* 7-15 min: intervalo de tiempo recomendado entre las dosis.

N. ${ }^{\circ}$ : número; ml: mililitros; mg: miligramos; U: unidades; /: por.

Tomado y adaptado de Audicana Berasategui MT y col..$^{51}$ 
International Consensus on drug allergy. Allergy. 2014; 69(4):420-37.

6. Giner Muñoz MT. Alergia a medicamentos. Conceptos básicos y actitud a seguir ante el pediatra. Protoc Diagn Ter Pediatr. 2013; 1:1-24.

7. Arriba-Méndez S. Novedades en el diagnóstico y tratamiento de la alergia a los medicamentos en los niños. Bol Pediatr. 2010; 50(212):87-92.

8. Cabello Martínez F. Alergológica 2005. Methodological aspects and sample chasracteristics of the study. J Investig Allergol Clin Immunol. 2009; 19(Suppl 2):2-6.

9. Chalhón IR. Reacciones adversas a los antibióticos $\beta$ lactámicos. En Jares J, Sánchez Borges M, De Falco A, Ivancevich J(eds.). Manual de Alergia a Medicamentos. Buenos Aires: AAAeIC-SLAAI; 2009; 6:54-66.

10. Parisi C. Alergia a Betalactámicos en pediatría, puesta al día y realidad en nuestro medio. IntraMed J. 2014;3(1):1-7.

11. Juri MC, Fernández Romero D, Larrauri B, Malbrán E, et al. Alergia a Drogas. Experiencia en 771 procedimientos. Buenos Aires. Medicina (B Aires). 2017; 77(3):180-4.

12. Ariza A, Mayorga C, Fernández TD, Barbero N, et al. Hypersensitivity reactions to beta-lactams: relevance of hapten-protein conjugates. J Investig Alergol Clin Immunol. 2015; 25(1):12-25.

13. MirakianR, LeechC,Krishna T, Richter A, etal.Management of allergy to penicillins and othrer beta-lactams. Clin Exp Allergy. 2015; 45(2):300-27.

14. Guzmán MA, Salinas J, Toche P, Afani A. Alergia a Betalactámicos. Rev Chil Infectol. 2004; 21(4):285-94.

15. Joint TaskForce on PracticeParameters; American Academy of Allergy, Asthma and Immunology; American College of Allergy, Asthma and Immunology; Joint Council of Allergy, Asthma and Immunology. Drug allergy: an updated practice parameter. Ann Allergy Asthma Immunol. 2010; 105(4):259-73

16. Blanca $M$. Allergic reactions to penicillins. A changing world? Allergy. 1995; 50(10):777-82.

17. Blanca M, Vega JM, García J, Miranda A, et al. New aspects of allergic reactions to betalactams: crossreactions and unique specificities. Clin Exp Allergy. 1994; 24(5):407-15.

18. Ponvert C, Weilnmann C, Wassenberg J, Walecki P, et al. Allergy to betalactam antibiotics in children: a prospective folow-up study in retreated children after negative responses in skin and challenge tests. Allergy. 2007; 62(1):426.

19. Hard D, Solensky R. Penicillin and Beta-lactam Hypersensitivity. Immunol Allergy Clin North Am. 2017; 37(4):643-62.

20. Macy E. Penicillin and Beta-lactam Allergy: Epidemiology and Diagnosis. Curr Allergy Asthma Rep. 2014; 14(11):476.

21. Fernández T, Mayorga $C$, Salas $M$, Barrionuevo E, et al. Evolution of diagnostic approaches in betalactam hypersensitivity. Expert Rev Clin Pharmacol.2017;10(6):67183.

22. Baldo BA, Zhao Z, Pham NH. Structural determinants of antibiotic allergy. Curr Allergy Rep. 2001; 1(1):23-31.

23. Romano A, Gaeta F, Arribas Poves MF, Valluzzi RL. Crossreactivity among beta-lactams. Curr Allergy Asthma Rep. 2016; 16(3):24.

24. Schnyder B, Brockow K. Pathogenesis of drug allergycurrent concepts and recent insights. Clin Exp Allergy. 2015; 45(9):1376-83.

25. Gerber BO, Pichler WJ. Cellular mechanisms of $\mathrm{T}$ cell mediated drug hypersensitivity. Curr Opin Immunol. 2004; 16(6):732-7.

26. Pichler WJ. Pharmacological interaction of drugs with antigen specific immune receptors: the $\mathrm{p}-\mathrm{i}$ concept. Curr Opin Allergy Clin Immunol. 2002; 2(4):301-5.
27. Pichler WJ, Adam J, Watkins S, Wuillemin N, et al. Drug hypersensitivity: how drugs stimulate $\mathrm{T}$ cells via pharmacological interaction with immune receptors. Int Arch Allergy Immunol. 2015; 168(1):13-24.

28. Pichler WJ, Hausmann O. Classification of drug hypersensitivity into allergic, $\mathrm{p}-\mathrm{i}$, and pseudo-allergic forms. Int Arch Allergy Immunol. 2016; 171(3-4):166-79.

29. HeelanK, Shear NH. Cutaneous Drug Reactionsin Children: an update. Paediatr Drugs. 2013; 15(6):493-503.

30. Legendre DP, Muzny CA, Marshall GD, Swiatlo E. Antibiotic hypersensitivity reactions and approaches to desensitization. Clin Infect Dis. 2014; 58(8):1140-8.

31. De Schryver SD, Netchiporouk E, Ben Shoshan M. Severe Serum Sickness-like Reaction:Challenges in Diagnosis and Management. J Clin Exp Dermatol Res. 2015; 6(3):279.

32. EinhornJ, Levis J.Dermatologic Diagnosis:Leukocytoclastic Vasculitis. Perm J. 2015; 19(3):77-8.

33. Mayorga C, Celik G, Rouzaire $P$, Whitaker $P$, et al. In vitro tests for drug hypersensitivity reactions: an ENDA/EAACI Drug Allergy Interest Group position paper. Allergy. 2016; 71(8):1103-34.

34. Belver MT, Michadavila A, Bobolea I, Feito M, et al. Severe delayed skin reactions related to drugs in the paediatric age group: A review of the subject by way of three cases (Stevens-Johnson syndrome, toxic epidermal necrolysis and DRESS). Allergol Immunopathol (Madr). 2016; 44(1):8395.

35. Cervini A, Buján M, Pierini A. Farmacodermias en la infancia. Dermatol Argent. 2011; 17(2):100-9.

36. Bell SJ, Pichler WJ. Penicillin-allergic patients react to penicillin-modified "self". Allergy.1989; 44(3):199-203.

37. Nagao-Dias AT, Teixeira FM, Coelho HL. Diagnosing immune-mediated reactions to drugs. Allergol Immunopathol (Madr). 2009; 37(2):98-104.

38. Abuaf N, RostaneH, Rajoely B, Gaouar H, etal. Comparison of two basophil activation markers CD63 and CD203c in the diagnosis of amoxicillin allergy. Clin Exp Allergy. 2008; 38(6):921-8

39. Sanz ML, Gamboa PM, Antépara I, Uasuf C, et al. Flow cytometric basophil activation test by detection of CD63 expression in patients with immediate-type reactions to betalactam antibiotics. Clin Exp Allergy. 2002; 32(2):277-86.

40. Fernández TD, Torres MJ, Blanca-LópezN, Rodríguez-Bada $\mathrm{JL}$, et al. Negativization rates of IgE radioimmunoassay and basophil activation test in immediate reactions to penicillins. Allergy. 2009; 64(2):242-8.

41. Longo N, Gamboa PM, Gastaminza G, Audicana MT, et al. Diagnosis of clavulanic acid allergy using basophil activation and leukotriene release by basophils. J Investig Allergol Clin Immunol. 2008; 18(6):473-5.

42. Salas M,Fernández-Santamaría R, Mayorga C, Barrionuevo E, et al. Use of the Basophil Activation Test May Reduce the Need for Drug Provocation in Amoxicillin-Clavulanic Allergy. J Allergy Clin Immunol Pract. 2018; 6(3):1010-8.

43. Blanca M, Pérez E, García J, Miranda A, et al. Anaphylaxis to amoxycillin but good tolerance for bencyl penicillin. In vivo and in vitro studies of specific IgE antibodies. Allergy. 1988; 43(7):508-10.

44. Macy E, Romano A, Khan D. Practical Management of Antibiotic Hypersensitivity in 2017. J Allergy Clin Immunol Pract. 2017; 5(3):577-86.

45. Rebello Gomes E, Fonseca j, Araujo L, Demoly P. Drug allergy claims in children: from self-reporting to confirmed diagnosis. Clin Exp Allergy. 2008; 38(1):191-8.

46. Ponvert C, Perrin Y, Bados-Albiero A, Le Bourgerois K, et al. Allergy to betalactam antibiotics in children: results of a 20-year study based on a clinical history, skin and challenge test. Pediatr Allergy Immunol. 2011; 22(4):411-8. 
47. Iammatteo M, Blumenthal KG, Saff R, Long A, et al. Safety and outcomes of test doses for the evaluation of adverse drug reactions: A 5 years retrospective review. J Allergy Clin Immunol Pract. 2014; 2(6):768-74.

48. VanArsdel PP Jr. Trastornos por hipersensibilidad. En Berkow R (ed.). El Manual Merck. 9. ${ }^{a}$ ed. Barcelona: Doyma; 1994.Págs.351-82.

49. Romero Valdez J, Pereira Q, Zini R, Canteros G. Reacciones de Hipersensibilidad. Rev Posgrado VIa Cátedra Med. 2007; 167:11-6.

50. Theofilopoulos A. The Raji, Conglutinin, and Anti-C3 assays for the detection of complement-fixing immune complexes. Methods Enzymol. 1981; 74 Pt C:511-30.

51. Audicana Berasategui MT, Ortega Rodríguez NR, García Ortega P, Uriel Villate O. Prevención y tratamiento de la alergia a los fármacos. Desensibilización. En Peláez Hernández A, Dávila González IJ (eds.). Tratado de alergología. Madrid: Ergón; 2007; 69:1369-75.

52. Schneck J, Fagot JP, Sekula P, Sassolas B, et al. Effects of treatments on the mortality of Stevens-Johnson syndrome and toxic epidermal necrolysis: A retrospective study on patients included in the prospective EuroSCAR Study. J Am Acad Dermatol. 2008; 58(1):33-40.

53. Sánchez-González MJ, Barbarroja-Escudero J, AntolínAmérigo D, Rodríguez-Rodríguez M. Reacciones alérgicas a fármacos. Medicine. 2013; 11(29):1808-18.
54. Baquero-Artigao F, Michavila A, Suárez-Rodríguez A, Hernandez A, et al. Documento de consenso de la Sociedad Española de Infectología Pediátrica, Sociedad Española de Inmunología Clínica y Alergia Pediátrica, Asociación Española dePediatría Extrahospitalaria y Atención Primara sobre antibioticoterapia en alergia a penicilina o amoxicilina. An Pediatr (Barc). 2017; 86(2):99.e1-9.

55. Pham MN, Ho HE, Desai M. Penicillin desensitization: Treatment of syphilis in pregnancy in penicillin-allergic patients. Ann Allergy Asthma Immunol. 2017; 118(5):537-41.

56. Cernadas JR, Brockow K, Romano A, Aberer W, et al. General considerations on rapid desensitization for drug hypersensitivity - a consensus statement. Allergy. 2010; 65(11):1357-66.

57. MacFadden DR, LaDelfa A, Leen J, Gold WL, et al. Impact of reported beta-lactam allergy on inpatient outcomes: a multicenter prospective cohort study. Clin Infect Dis. 2016; 63(7):904-10.

58. Norton AE, Konvinse K, Phillips EJ, Broyles AD. Antibiotic Allergy in Pediatrics. Pediatrics. 2018; 141(5):e20172497.

59. De las Vecillas Sánchez L, Alenazy L, García-Neuer M, Castells M. Drug Hypersensitivity and Desensitizations: Mechanisms and New Approaches. Int J Mol Sci. 2017; 18(6):e1316 